\title{
Rapid Detection of Salmonella Enteritidis, Typhimurium, and Thompson by Specific Peak Analysis Using Matrix-Assisted Laser Desorption Ionization Time-of-Flight Mass Spectrometry
}

\author{
Seung-Min Yang ${ }^{1,+}$, Eiseul Kim ${ }^{1,+}$, Dayoung Kim ${ }^{1}$, Jiwon Baek ${ }^{2}$, Hyunjin Yoon ${ }^{2} \mathbb{D}$ and Hae-Yeong Kim ${ }^{1, *(\mathbb{D})}$ \\ 1 Institute of Life Sciences \& Resources and Department of Food Science and Biotechnology, \\ Kyung Hee University, Yongin 17104, Korea; ysm9284@gmail.com (S.-M.Y.); eskim89@khu.ac.kr (E.K.); \\ ekdudvv4589@naver.com (D.K.) \\ 2 Department of Molecular Science and Technology, Ajou University, Suwon 16499, Korea; \\ qorwldnjs78@ajou.ac.kr (J.B.); yoonh@ajou.ac.kr (H.Y.) \\ * Correspondence: hykim@khu.ac.kr; Tel.: +82-31-201-2600; Fax: +82-31-204-8116 \\ + These authors contributed equally to the work.
}

\section{check for} updates

Citation: Yang, S.-M.; Kim, E.; Kim, D.; Baek, J.; Yoon, H.; Kim, H.-Y.

Rapid Detection of Salmonella Enteritidis, Typhimurium, and Thompson by Specific Peak Analysis Using Matrix-Assisted Laser Desorption Ionization Time-of-Flight Mass Spectrometry. Foods 2021, 10, 933. https://doi.org/10.3390/ foods10050933

Academic Editor: Clara Sousa

Received: 20 March 2021

Accepted: 21 April 2021

Published: 23 April 2021

Publisher's Note: MDPI stays neutral with regard to jurisdictional claims in published maps and institutional affiliations.

Copyright: (c) 2021 by the authors. Licensee MDPI, Basel, Switzerland. This article is an open access article distributed under the terms and conditions of the Creative Commons Attribution (CC BY) license (https:// creativecommons.org/licenses/by/ $4.0 /)$.

\begin{abstract}
Rapid detection of Salmonella serovars is important for the effective control and monitoring of food industries. In this study, we evaluate the application of matrix-assisted laser desorption/ionization time-of-flight mass spectrometry for the rapid detection of three serovars, Enteritidis, Typhimurium, and Thompson, that are epidemiologically important in Korea. All strains were identified at the genus level, with a mean score of 2.319 using the BioTyper database, and their protein patterns were confirmed to be similar by principal component analysis and main spectrum profile dendrograms. Specific peaks for the three serovars were identified by analyzing 65 reference strains representing 56 different serovars. Specific mass peaks at $3018 \pm 1$ and $6037 \pm 1,7184 \pm 1$, and $4925 \pm 1 \mathrm{~m} / \mathrm{z}$ were uniquely found in the reference strains of serovars Enteritidis, Typhimurium, and Thompson, respectively, and they showed that the three serovars can be differentiated from each other and 53 other serovars. We verified the reproducibility of these mass peaks in 132 isolates, and serovar classification was achieved with $100 \%$ accuracy when compared with conventional serotyping through antisera agglutination. Our method can rapidly detect a large number of strains; hence, it will be useful for the high-throughput screening of Salmonella serovars.
\end{abstract}

Keywords: Salmonella serotyping; MALDI-TOF MS; identification; detection; specific peak; Enteritidis; Typhimurium; Thompson

\section{Introduction}

Salmonella, one of the main causes of foodborne diseases, is a significant public health concern. Salmonella is known to be spread primarily through the consumption of contaminated food or water [1-3]. Salmonella infection can cause serious illness in humans, especially infants and the elderly, causing diseases such as typhoid fever and gastroenteritis and even leading to death [4]. It is estimated that about 1.2 million cases of Salmonella infection occur annually in the United States alone, and Salmonella has the second-highest rate of incidence among the pathogenic bacteria that cause food poisoning in Korea [5,6]. The genus Salmonella is divided into two species: S. bongori and S. enterica. S. enterica is further subdivided into six subspecies [7]. Of these, S. enterica subsp. enterica is of clinical relevance and is the most frequently isolated subspecies [7,8]. Salmonella serotyping according to the White-Kauffmann-Le Minor scheme is based on a combination of the biochemical identification of somatic $\mathrm{O}$, flagella $\mathrm{H}$, and capsular Vi antigens and is recognized worldwide as a gold standard for differentiation below the subspecies level [7,9]. 
Although more than 2600 serovars exist, Enteritidis and Typhimurium are the major serovars of foodborne infection in humans and are considered very important in public health [10]. Serovar Enteritidis is the most common serovar of infection of humans worldwide [11]. According to the World Health Organization, serovar Enteritidis is the most common cause of gastroenteritis, although previously, serovar Typhimurium was the main cause [10]. Recently, a large-scale outbreak of gastroenteritis caused by serovar Thompson was reported in Korea, with a total of 2207 people infected [12]. Epidemiological and traceback evidence showed that egg whites used to make a chocolate cake were infected with Thompson, and the chocolate cake was announced as the source of infection [12]. In order to ensure the safety of food, regulations on the microbial inspection standards of edible eggs were revised and adjusted in 2019 so that Enteritidis, Typhimurium, and Thompson are not found in food. Thus, timely detection of the three prominent serovars is very important.

Detection of the causative pathogen is usually an essential step in epidemiological investigation [4]. Unfortunately, serotyping, typically performed by slide agglutination, is time-intensive, costly, complicated, and laborious and requires more than 150 specific antisera $[7,13]$. This method also requires that workers have the expertise necessary to interpret the aggregation results. Biochemical tests and morphological descriptions may produce ambiguous results [4]. Although whole-genome sequencing is becoming ever more accessible and is useful for serotyping because it provides comprehensive genetic information, it is not practical for sequencing multiple isolates simultaneously [4] However, matrix-assisted laser desorption ionization time-of-flight mass spectrometry (MALDI-TOF MS) is a powerful tool that can detect bacterial strains rapidly and accurately. This technique is increasingly being used for the detection of bacterial strains [14-18]. While most of these studies have demonstrated the usefulness of the technique for species or subspecies identification, bacterial detection below the subspecies level, such as the discrimination of serovars, has rarely been addressed [7].

In this study, we evaluate the performance of MALDI-TOF MS for the detection of major Salmonella serovars isolated from various food samples, including egg white, and discover peaks specific to serovars Enteritidis, Typhimurium, and Thompson by analyzing the mass spectra of Salmonella serovars. The accuracy of specific peaks from the MALDI-TOF MS spectra was confirmed using conventional serotyping through antisera agglutination.

\section{Materials and Methods}

\subsection{Isolation of Salmonella Strains}

Reference strains of 12 Enteritidis, Typhimurium, and Thompson Salmonella serovars and 53 other serovars used in this study are presented in Table 1. Salmonella strains were isolated from various sources, such as processed food, fresh food, chicken meat, vegetables, egg white, and livestock. These strains were isolated as recommended by the FDA [19]. One milliliter of sample filtrate was pre-enriched by inoculation into $9 \mathrm{~mL}$ of buffered peptone water (Difco, Becton \& Dickinson, Sparks, MD, USA), and the culture was incubated at $37^{\circ} \mathrm{C}$ for $18 \mathrm{~h}$. The cultured cells were inoculated into $10 \mathrm{~mL}$ of Rappaport-Vassiliadis broth (Difco) and incubated at $42{ }^{\circ} \mathrm{C}$ for $24 \mathrm{~h}$. The inoculum was streaked on xylose lysine desoxycholate agar (XLD, Difco) and incubated at $37^{\circ} \mathrm{C}$ for $24 \mathrm{~h}$ under aerobic conditions. Then, a colony suspected of being Salmonella was screened on XLD agar. All reference strains and isolates were cultured on tryptic soy agar (Difco) at $37^{\circ} \mathrm{C}$ for $18 \mathrm{~h}$ under aerobic conditions and then subcultured in tryptic soy broth for storage in $30 \%$ glycerol $(v / v)$ at $-80^{\circ} \mathrm{C}$. 
Table 1. List of Salmonella reference strains used in this study.

\begin{tabular}{|c|c|}
\hline Serovars & Designated As: \\
\hline Salmonella Enteritidis & MFDS $^{1}$ 1004839, MFDS 1008613, MFDS 1009945, MFDS 1010897, KCA ${ }^{2}$ 57, KCA 65 \\
\hline Salmonella Typhimurium & ATCC $^{3}$ 14028, ATCC 19585, MFDS 1000002, MFDS 1000030 \\
\hline Salmonella Thompson & CCARM $^{4} 8530$, MFDS 1004024 \\
\hline Salmonella Aberdeen & $\mathrm{NCCP}^{5} 10142$ \\
\hline Salmonella Agona & MFDS 1004876 \\
\hline Salmonella Albany & MFDS K000014 \\
\hline Salmonella Anatum & $\mathrm{KVCC}^{6} \mathrm{BA} 0000586$ \\
\hline Salmonella Bardo & NCCP 13572 \\
\hline Salmonella Bareilly & NCCP 16324 \\
\hline Salmonella Berta & KVCC BA0000581 \\
\hline Salmonella Blockley & NCCP 10769 \\
\hline Salmonella Bovismorbificans & NCCP 12244 \\
\hline Salmonella Braenderup & MFDS 1008393 \\
\hline Salmonella Brandenburg & NCCP 12835 \\
\hline Salmonella Cerro & NCCP 12215 \\
\hline Salmonella Choleraesuis & ATCC 13312 \\
\hline Salmonella Derby & MFDS 1009813 \\
\hline Salmonella Dessau & MFDS 1010078 \\
\hline Salmonella Elisabethville & NCCP 14030 \\
\hline Salmonella Gallinarum & ATCC 9120 \\
\hline Salmonella Give & NCCP 13696 \\
\hline Salmonella Hadar & NCCP 13571 \\
\hline Salmonella Heidelberg & NCCP 11693 \\
\hline Salmonella Hindmarsh & BFR $^{7} 12$ \\
\hline Salmonella I 4,[5],12:i:- & MFDS 1004858 \\
\hline Salmonella Infantis & MFDS 1010567 \\
\hline Salmonella Javiana & $\mathrm{FDA}^{8} 05$ \\
\hline Salmonella Kedougou & NCCP 11685 \\
\hline Salmonella Kentucky & NCCP 11686 \\
\hline Salmonella Kottbus & NCCP 12234 \\
\hline Salmonella Litchfield & FDA 33 \\
\hline Salmonella Livingstone & MFDS 1004819 \\
\hline Salmonella London & MFDS 1004861 \\
\hline Salmonella Madelia & FDA 30 \\
\hline Salmonella Mbandaka & KVCC BA1800001 \\
\hline Salmonella Meleagridis & FDA 34 \\
\hline Salmonella Minnesota & MFDS 1008449 \\
\hline Salmonella Mississippi & FDA 32 \\
\hline Salmonella Montevideo & MFDS 1006814 \\
\hline Salmonella Muenchen & KCA 03 \\
\hline Salmonella Muenster & FDA 23 \\
\hline Salmonella Newington & NCCP 10894 \\
\hline Salmonella Newport & MFDS 1005422 \\
\hline Salmonella Panama & MFDS 1004857 \\
\hline Salmonella Paratyphi A & NCCP 14759 \\
\hline Salmonella Paratyphi B & ATCC 10719 \\
\hline Salmonella Paratyphi C & ATCC 13428 \\
\hline Salmonella Poona & FDA 22 \\
\hline Salmonella Reading & MFDS 1007899 \\
\hline Salmonella Rissen & NCCP 13709 \\
\hline Salmonella Schwarzengrund & MFDS 1006893 \\
\hline Salmonella Senftenberg & CCARM 0041 \\
\hline Salmonella Singapore & NCCP 12218 \\
\hline Salmonella Vinohrady & NCCP 12217 \\
\hline Salmonella Virchow & MFDS 1004870 \\
\hline Salmonella Weltevreden & NCCP 12239 \\
\hline
\end{tabular}

${ }^{1}$ MFDS, Ministry of Food \& Drug Safety; ${ }^{2}$ KCPB, Korea Consumer Protection Board; ${ }^{3}$ ATCC, American Type Culture Collection; ${ }^{4}$ CCARM, Culture Collection of Antibiotics Resistant Microbes; ${ }^{5} \mathrm{NCCP}$, National Culture Collection for Pathogens; ${ }^{6} \mathrm{KVCC}$, Korea Veterinary Culture Collection; ${ }^{7} \mathrm{BFR}$, Federal Institute for Risk Assessment; ${ }^{8}$ FDA, US Food and Drug Administration. 


\subsection{Identification of Serovar-Specific Peaks Using MALDI-TOF MS}

Proteins of Salmonella strains were extracted using an ethanol/formic acid extraction method [14,15]. A loopful of Salmonella cells was suspended in $300 \mu \mathrm{L}$ deionized water, and $900 \mu \mathrm{L}$ of ethanol was added. The cell suspension was centrifuged at $13,600 \times g$ for $5 \mathrm{~min}$, and the pellet was dried at room temperature. The dried pellet was resuspended in $50 \mu \mathrm{L}$ of $70 \%$ formic acid and $50 \mu \mathrm{L}$ of acetonitrile and centrifugation at $13,600 \times g$ for $5 \mathrm{~min}$. Then, $1 \mu \mathrm{L}$ of supernatant was spotted onto an MSP 96 polished steel target plate (Bruker Daltonics, Bremen, Germany) and allowed to dry at room temperature. After drying, each spot was overlaid with $1 \mu \mathrm{L}$ of matrix solution ( $\alpha$-cyano-4-hydroxycinnamic acid in $50 \%$ acetonitrile and $2.5 \%$ trifluoroacetic acid). The acquisition of mass spectra was performed using a Microflex LT bench-top mass spectrometer (Bruker Daltonics). The mass spectra were measured using FlexControl software, with the default parameter settings, for bacterial identification. Calibration of the instrument was performed using a bacterial test standard (Bruker Daltonics) consisting of Escherichia coli protein extract. The obtained mass spectra were analyzed for the identification of serovar-specific peaks, as identified in previous studies $[16,20,21]$. Before identifying the specific peaks, the raw spectra were preprocessed by normalizing, smoothing, and baseline subtraction [22]. Spectra of poor quality, such as peaks with significant background noise or very low intensity, were excluded. Serovar-specific peaks were determined by comparing the spectra for different serovars using FlexAnalysis software version 3.4 (Bruker Daltonics).

\subsection{Identification of Serovars by BioTyper and Specific Peaks}

For the detection of Salmonella isolates, proteins of strains were extracted using a direct transfer method [15]. A fresh colony was smeared on an MSP 96 polished steel target plate (Bruker Daltonics) using a toothpick and overlaid with $1 \mu \mathrm{L}$ matrix solution. After drying, the target plate was loaded into a Microflex LT bench-top mass spectrometer, which includes BioTyper database version 3.4 (5627 database entries), under the same conditions described above. The results were presented as a score between 0 to 3 . The score interpretation of the four categories was as follows: a score of $\geq 2.3$ indicates a high level of probability of species, scores between 2.000-2.299 indicate probable species identification, scores between 1.700-1.999 indicate probable genus identification, and a score $<1.7$ indicates no reliable identification. To identify serovars with specific peaks, raw spectra were normalized, and the peak intensity and areas of the isolates were obtained using FlexAnalysis software (Bruker Daltonics). Salmonella serovars were determined by identifying the presence or absence of serovar-specific peaks. Main spectrum profile (MSP) dendrograms and principal component analysis (PCA) for all strains were conducted as per the standard operating procedure using MALDI BioTyper software version 3.1 (Bruker Daltonics). The mean peak masses, peak frequencies, and peak intensities were visualized.

\subsection{Serotyping through the Agglutination of Antisera}

The serotypes of the isolates used in this study were confirmed using a traditional serotyping method, as described in a previous study [1], and compared with the results of MALDI-TOF MS. Serology was performed using slide agglutination tests with commercial monovalent or polyvalent somatic $\mathrm{O}$ and flagellar $\mathrm{H}$ antisera (Difco), according to the manufacturer's instructions. Serovars of isolates were determined using antigenic formulae based on the White-Kauffmann-Le Minor scheme [9]. 


\section{Results}

\subsection{Isolation and Identification of Salmonella Strains}

In the food and livestock samples, presumptive Salmonella colonies were grown on XLD agar and identified using MALDI-TOF MS with the BioTyper database. One hundred and thirty-two colonies were identified as Salmonella species. Salmonella was isolated from processed food (31 strains), fresh food (19 strains), chicken meat (45 strains), vegetables (18 strains), egg white (2 strains), and livestock (17 strains) (Table 2). Of these isolates, 88 strains $(66.67 \%)$ were identified at the highly probable species level (log score $\geq 2.3$ ) and 44 strains $(33.33 \%$ ) were identified at the probable species level (log score of 2.0-2.3). All isolates were identified as Salmonella species at the genus level by MALDI-TOF MS with the BioTyper database.

Table 2. List of isolates identified as Salmonella species by MALDI-TOF MS.

\begin{tabular}{cc}
\hline Source of Sample & Strains (Number of Isolates) \\
\hline Processed food & S1-S16, S23-S24, S56-S67, S88 $(n=31)$ \\
Fresh food & S27, S37-S49, S76, S89, S126-S127, S132 $(n=19)$ \\
Chicken meat & S30-S36, S50-S55, S90-S121 $(n=45)$ \\
Vegetable & S68-S75, S77-S86 $(n=18)$ \\
Egg white & S130-S131 $(n=2)$ \\
Livestock & S17-S22, S25-S26, S28-S29, S87, S122-S125, S128-S129 $(n=17)$ \\
\hline
\end{tabular}

In total, 12 reference strains and 132 isolates were analyzed by MALDI-TOF MS to evaluate the robustness of the BioTyper database. MSP dendrograms and PCA clustering are useful for distinguishing between closely related strains and determining associations between the strains [22]. These methods were used to confirm the discriminative power of mass peak analysis for serovar detection. All strains of serovars Enteritidis, Typhimurium, and Thompson fell into three distinct groups in the dendrogram (Figure 1). The first cluster contained serovar Enteritidis, the second cluster contained Typhimurium, and the third cluster included all three serovars-Enteritidis, Typhimurium, and Thompson. PCA was used to cluster specimens according to their relative intensities and mass values (Figure 2). This clustering showed that the three serovars were not clearly separated. Additionally, a difference in mass peak pattern according to the source of isolation was not observed. Therefore, both the MSP dendrogram and PCA confirmed that the mass spectra of the three serovars were similar. 




Figure 1. Main spectrum profile (MSP) dendrogram generated based on $m / z$ values and relative intensities of 12 reference strains and 132 isolates. Bold indicates reference strains. 
A

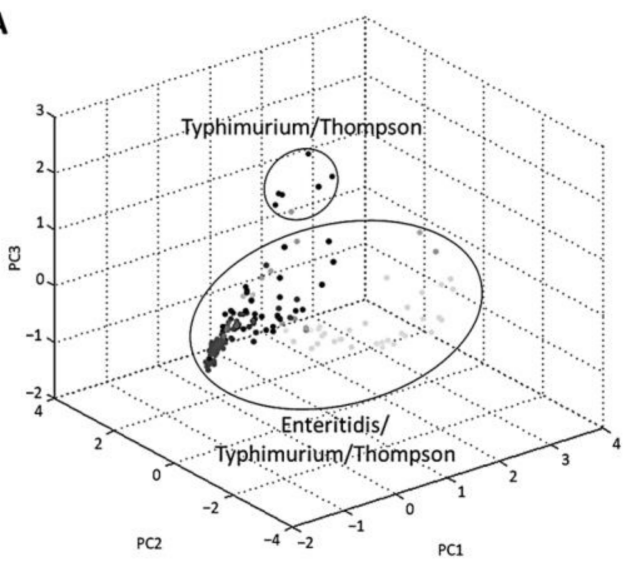

B

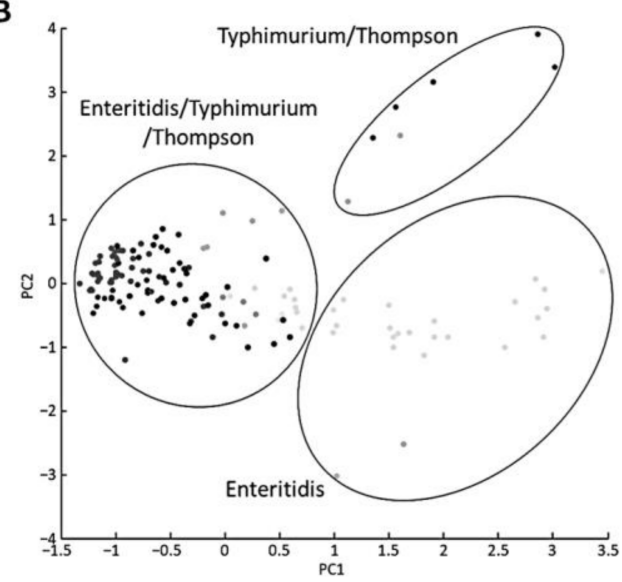

Figure 2. Principal component analysis (PCA) generated by mass spectra of 12 reference strains and 132 isolates. Each dot on the (A) three-dimensional plot and (B) two-dimensional plot represents strains.

\subsection{Analysis of Serovar-Specific Peaks}

The mass spectrum profiles of Salmonella Enteritidis, Typhimurium, and Thompson showed similar patterns (Figure 3). The discriminative ability at the serovar level was evaluated by analyzing multiple mass spectra obtained from 53 different serovars. A total of 499 mass peaks were extracted from the spectrum profiles of 12 reference strains of serovars Enteritidis, Typhimurium, and Thompson and analyzed for peak value and intensity. In this process, one peak was excluded due to poor quality spectra with low intensity (36.189 arbitrary units). The peaks from these serovars were compared with 770 peaks extracted from 53 other serovar strains to confirm that they were unique peaks not found in other serovars.

Table 3. Presence/absence of specific peaks for 12 reference strains.

\begin{tabular}{ccccc}
\hline Source of Sample & \multicolumn{5}{c}{ Mass Peak $(\mathbf{m} / \mathbf{z})^{\mathbf{1}}$} \\
\cline { 2 - 5 } & $\mathbf{3 0 1 8} \pm \mathbf{1}$ & $\mathbf{6 0 3 7} \pm \mathbf{1}$ & $\mathbf{7 1 8 4} \pm \mathbf{1}$ & $\mathbf{4 9 2 5} \pm \mathbf{1}$ \\
\hline Enteritidis MFDS 1004839 & + & + & - & - \\
Enteritidis MFDS 1008613 & + & + & - & - \\
Enteritidis MFDS 1009945 & + & + & - & - \\
Enteritidis MFDS 1010897 & + & + & - & - \\
Enteritidis KCA 57 & + & + & - & - \\
Enteritidis KCA 65 & + & + & - & - \\
Typhimurium ATCC 14028 & - & - & + & - \\
Typhimurium ATCC 19585 & - & - & + & - \\
Typhimurium MFDS 1000002 & - & - & + & + \\
Typhimurium MFDS 1000030 & - & - & + & + \\
Thompson CCARM 8530 & - & - & - & - \\
Thompson MFDS 1004024 & - & - & - & - \\
\hline
\end{tabular}

1, presence of peak; -, absence of peak. 




Figure 3. MALDI-TOF MS spectra of reference strains of serovars Enteritidis, Typhimurium, and Thompson; $m / z$, mass-tocharge ratio; a.u., arbitrary units. 
Table 4. Presence/absence of specific peaks for 20 other pathogenic bacterial strains.

\begin{tabular}{|c|c|c|c|c|}
\hline \multirow{2}{*}{ Strains } & \multicolumn{4}{|c|}{ Mass Peak $(m / z)$} \\
\hline & $3018 \pm 1$ & $6037 \pm 1$ & $7184 \pm 1$ & $4925 \pm 1$ \\
\hline Escherichia coli NCCP 14039 & - & - & - & - \\
\hline Escherichia coli NCCP 14037 & - & - & - & - \\
\hline Escherichia coli NCCP 14033 & - & - & - & - \\
\hline Escherichia coli MFDS 0064 & - & - & - & - \\
\hline Escherichia coli MFDS 5919 & - & - & - & - \\
\hline Escherichia coli O157:H7 MFDS 43894 & - & - & - & - \\
\hline Escherichia coli O157:H7 ATCC 43890 & - & - & - & - \\
\hline Staphylococcus aureus KCTC ${ }^{1} 12113$ & - & - & - & - \\
\hline Staphylococcus aureus KCTC 1928 & - & - & - & - \\
\hline Staphylococcus aureus ATCC 25923 & - & - & - & - \\
\hline Bacillus cereus ATCC 10876 & - & - & - & - \\
\hline Bacillus cereus KCCM ${ }^{2} 1174$ & - & - & - & - \\
\hline Listeria monocytogenes КСТС 3569 & - & - & - & - \\
\hline Listeria ivanovii ATCC 19119 & - & - & - & - \\
\hline Vibrio cholerae ATCC 14033 & - & - & - & - \\
\hline Vibrio cholerae ATCC 14035 & - & - & - & - \\
\hline Shigella flexneri KCTC 2517 & - & - & - & - \\
\hline Shigella sonnei КСТС 2518 & - & - & - & - \\
\hline Cronobacter sakazakii ATCC 29544 & - & - & - & - \\
\hline Citrobacter freundii ATCC 8090 & - & - & - & - \\
\hline
\end{tabular}

${ }^{1}$ KCTC, Korean Collection for Type Cultures; ${ }^{2}$ KCCM, Korean Culture Center of Microorganisms.

\subsection{Identification of Isolates by Serovar-Specific Peaks}

To validate our approach to the detection of Enteritidis, Typhimurium, and Thompson, the most epidemiologically important serovars, 132 Salmonella isolates were analyzed using MALDI-TOF MS. Serovar-specific peaks for Salmonella Enteritidis, Typhimurium, and Thompson were identified by the mass peaks described above (Figure 4). A total of 132 isolates were identified using these mass peaks to confirm that these peaks, identified in the reference strains, were present in many isolates. In total, 55 isolates were identified as serovar Enteritidis by peak analysis (Table 5), while 74 isolates were identified as serovar Typhimurium and 3 as Thompson. The mass peak at $3018 \pm 1$ and $6037 \pm 1 \mathrm{~m} / \mathrm{z}$, specific to serovar Enteritidis, was present in all 55 isolates, and other serovar-specific mass peaks were absent in these isolates. The mass peak at $7184 \pm 1 \mathrm{~m} / \mathrm{z}$, specific to serovar Typhimurium, was present in 74 isolates. In these strains, the specific peaks for Enteritidis and Thompson were absent. The mass peak at $4925 \pm 1 \mathrm{~m} / \mathrm{z}$ was present in all isolates identified as serovar Thompson, whereas this mass peak was absent in serovar Enteritidis and Typhimurium. Therefore, the three mass peaks were considered to be specific peaks that could be used to detect the serovars Enteritidis, Typhimurium, and Thompson. 
A

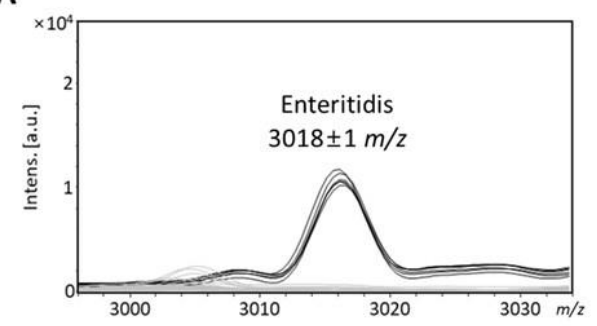

C

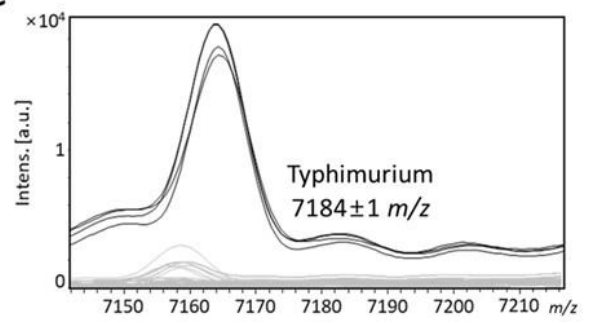

B

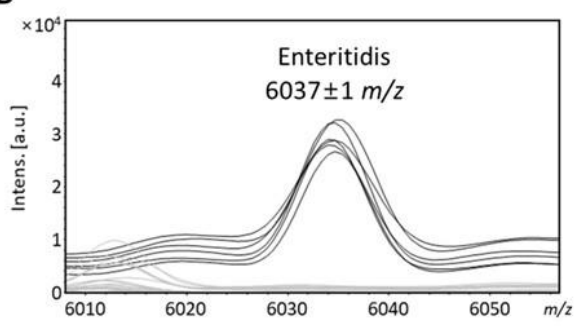

D

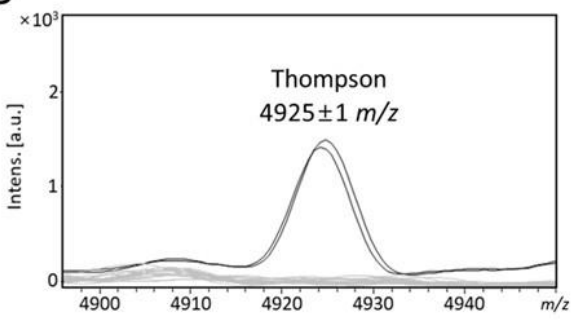

Figure 4. Specific peaks for three serovars. (A) Mass peak at $3018 \pm 1 \mathrm{~m} / \mathrm{z}$ presents in serovar Enteritidis reference strains, (B) mass peak at $6037 \pm 1 \mathrm{~m} / \mathrm{z}$ presents in serovar Enteritidis strains, (C) mass peak at $7184 \pm 1 \mathrm{~m} / \mathrm{z}$ presents in serovar Typhimurium strains, and (D) mass peak at $4925 \pm 1 \mathrm{~m} / \mathrm{z}$ presents in serovar Thompson.

Table 5. Identification of isolates by antisera agglutination and specific peaks.

\begin{tabular}{|c|c|c|c|c|}
\hline \multirow{2}{*}{ Strains } & \multirow{2}{*}{ Source } & \multirow{2}{*}{ Serovars ${ }^{1}$} & \multicolumn{2}{|c|}{ MALDI-TOF MS } \\
\hline & & & BioTyper & Specific Peak \\
\hline S1 & Processed food & Enteritidis & Salmonella sp. & Enteritidis $(3017.9,6038.4 \mathrm{~m} / \mathrm{z})$ \\
\hline $\mathrm{S} 2$ & Processed food & Enteritidis & Salmonella sp. & Enteritidis $(3017.9,6038.0 \mathrm{~m} / \mathrm{z})$ \\
\hline S3 & Processed food & Enteritidis & Salmonella sp. & Enteritidis $(3017.9,6038.3 \mathrm{~m} / \mathrm{z})$ \\
\hline $\mathrm{S} 4$ & Processed food & Enteritidis & Salmonella sp. & Enteritidis $(3017.9,6038.5 \mathrm{~m} / \mathrm{z})$ \\
\hline S5 & Processed food & Enteritidis & Salmonella sp. & Enteritidis $(3017.9,6038.3 \mathrm{~m} / \mathrm{z})$ \\
\hline S6 & Processed food & Enteritidis & Salmonella sp. & Enteritidis $(3017.8,6038.6 \mathrm{~m} / \mathrm{z})$ \\
\hline S7 & Processed food & Enteritidis & Salmonella sp. & Enteritidis $(3018.0,6038.2 \mathrm{~m} / \mathrm{z})$ \\
\hline S8 & Processed food & Enteritidis & Salmonella sp. & Enteritidis $(3018.3,6038.2 \mathrm{~m} / \mathrm{z})$ \\
\hline S9 & Processed food & Enteritidis & Salmonella sp. & Enteritidis $(3018.2,6038.6 \mathrm{~m} / \mathrm{z})$ \\
\hline S10 & Processed food & Enteritidis & Salmonella sp. & Enteritidis $(3018.5,6039.2 \mathrm{~m} / \mathrm{z})$ \\
\hline S11 & Processed food & Enteritidis & Salmonella sp. & Enteritidis $(3018.0,6038.3 \mathrm{~m} / \mathrm{z})$ \\
\hline S12 & Processed food & Enteritidis & Salmonella sp. & Enteritidis $(3018.3,6038.8 \mathrm{~m} / \mathrm{z})$ \\
\hline S13 & Processed food & Enteritidis & Salmonella sp. & Enteritidis $(3017.5,6037.5 \mathrm{~m} / \mathrm{z})$ \\
\hline S14 & Processed food & Enteritidis & Salmonella sp. & Enteritidis $(3017.7,6037.7 \mathrm{~m} / \mathrm{z})$ \\
\hline S15 & Processed food & Enteritidis & Salmonella sp. & Enteritidis $(3018.0,6038.5 \mathrm{~m} / \mathrm{z})$ \\
\hline S16 & Processed food & Enteritidis & Salmonella sp. & Enteritidis $(3017.9,6038.1 \mathrm{~m} / \mathrm{z})$ \\
\hline S17 & Livestock & Enteritidis & Salmonella sp. & Enteritidis $(3017.9,6038.0 \mathrm{~m} / \mathrm{z})$ \\
\hline S18 & Livestock & Enteritidis & Salmonella sp. & Enteritidis $(3017.9,6038.1 \mathrm{~m} / \mathrm{z})$ \\
\hline S19 & Livestock & Enteritidis & Salmonella sp. & Enteritidis $(3018.1,6038.6 \mathrm{~m} / \mathrm{z})$ \\
\hline S20 & Livestock & Enteritidis & Salmonella sp. & Enteritidis $(3017.9,6038.3 \mathrm{~m} / \mathrm{z})$ \\
\hline $\mathrm{S} 21$ & Livestock & Enteritidis & Salmonella sp. & Enteritidis $(3018.0,6038.3 \mathrm{~m} / \mathrm{z})$ \\
\hline
\end{tabular}


Table 5. Cont.

\begin{tabular}{|c|c|c|c|c|}
\hline \multirow{2}{*}{ Strains } & \multirow{2}{*}{ Source } & \multirow{2}{*}{ Serovars $^{1}$} & \multicolumn{2}{|c|}{ MALDI-TOF MS } \\
\hline & & & BioTyper & Specific Peak \\
\hline S22 & Livestock & Enteritidis & Salmonella sp. & Enteritidis $(3018.2,6038.5 \mathrm{~m} / \mathrm{z})$ \\
\hline $\mathrm{S} 23$ & Processed food & Enteritidis & Salmonella sp. & Enteritidis $(3018.1,6038.4 \mathrm{~m} / \mathrm{z})$ \\
\hline S24 & Processed food & Enteritidis & Salmonella sp. & Enteritidis $(3018.0,6038.2 \mathrm{~m} / \mathrm{z})$ \\
\hline S25 & Livestock & Enteritidis & Salmonella sp. & Enteritidis $(3017.7,6037.4 \mathrm{~m} / \mathrm{z})$ \\
\hline S26 & Livestock & Enteritidis & Salmonella sp. & Enteritidis $(3017.7,6037.6 \mathrm{~m} / \mathrm{z})$ \\
\hline $\mathrm{S} 27$ & Fresh food & Enteritidis & Salmonella sp. & Enteritidis $(3017.8,6038.0 \mathrm{~m} / \mathrm{z})$ \\
\hline S28 & Livestock & Enteritidis & Salmonella sp. & Enteritidis $(3017.8,6038.1 \mathrm{~m} / \mathrm{z})$ \\
\hline S29 & Livestock & Enteritidis & Salmonella sp. & Enteritidis $(3018.0,6038.2 \mathrm{~m} / \mathrm{z})$ \\
\hline S30 & Chicken meat & Enteritidis & Salmonella sp. & Enteritidis $(3018.0,6038.7 \mathrm{~m} / \mathrm{z})$ \\
\hline S31 & Chicken meat & Enteritidis & Salmonella sp. & Enteritidis $(3017.9,6038.3 \mathrm{~m} / \mathrm{z})$ \\
\hline S32 & Chicken meat & Enteritidis & Salmonella sp. & Enteritidis $(3018.0,6038.2 \mathrm{~m} / \mathrm{z})$ \\
\hline $\mathrm{S} 33$ & Chicken meat & Enteritidis & Salmonella sp. & Enteritidis $(3018.8,6038.4 \mathrm{~m} / \mathrm{z})$ \\
\hline S34 & Chicken meat & Enteritidis & Salmonella sp. & Enteritidis $(3018.1,6038.8 \mathrm{~m} / \mathrm{z})$ \\
\hline S35 & Chicken meat & Enteritidis & Salmonella sp. & Enteritidis $(3018.7,6037.9 \mathrm{~m} / \mathrm{z})$ \\
\hline S36 & Chicken meat & Enteritidis & Salmonella sp. & Enteritidis $(3017.9,6038.1 \mathrm{~m} / \mathrm{z})$ \\
\hline S37 & Fresh food & Enteritidis & Salmonella sp. & Enteritidis $(3018.0,6038.5 \mathrm{~m} / \mathrm{z})$ \\
\hline S38 & Fresh food & Enteritidis & Salmonella sp. & Enteritidis $(3018.1,6038.6 \mathrm{~m} / \mathrm{z})$ \\
\hline S39 & Fresh food & Enteritidis & Salmonella sp. & Enteritidis $(3018.1,6038.8 \mathrm{~m} / \mathrm{z})$ \\
\hline S40 & Fresh food & Enteritidis & Salmonella sp. & Enteritidis $(3018.0,6038.5 \mathrm{~m} / \mathrm{z})$ \\
\hline S41 & Fresh food & Enteritidis & Salmonella sp. & Enteritidis $(3018.1,6038.9 \mathrm{~m} / \mathrm{z})$ \\
\hline S42 & Fresh food & Enteritidis & Salmonella sp. & Enteritidis $(3018.2,6038.8 \mathrm{~m} / \mathrm{z})$ \\
\hline $\mathrm{S} 43$ & Fresh food & Enteritidis & Salmonella sp. & Enteritidis $(3017.6,6037.5 \mathrm{~m} / \mathrm{z})$ \\
\hline S44 & Fresh food & Enteritidis & Salmonella sp. & Enteritidis $(3017.8,6038.0 \mathrm{~m} / \mathrm{z})$ \\
\hline $\mathrm{S} 45$ & Fresh food & Enteritidis & Salmonella sp. & Enteritidis $(3018.0,6038.3 \mathrm{~m} / \mathrm{z})$ \\
\hline S46 & Fresh food & Enteritidis & Salmonella sp. & Enteritidis $(3017.9,6038.0 \mathrm{~m} / \mathrm{z})$ \\
\hline S47 & Fresh food & Enteritidis & Salmonella sp. & Enteritidis $(3017.9,6038.2 \mathrm{~m} / \mathrm{z})$ \\
\hline S48 & Fresh food & Enteritidis & Salmonella sp. & Enteritidis $(3018.1,6038.4 \mathrm{~m} / \mathrm{z})$ \\
\hline S49 & Fresh food & Enteritidis & Salmonella sp. & Enteritidis $(3018.3,6038.8 \mathrm{~m} / \mathrm{z})$ \\
\hline S50 & Chicken meat & Enteritidis & Salmonella sp. & Enteritidis $(3018.0,6038.4 \mathrm{~m} / \mathrm{z})$ \\
\hline S51 & Chicken meat & Enteritidis & Salmonella sp. & Enteritidis $(3018.2,6037.6 \mathrm{~m} / \mathrm{z})$ \\
\hline S52 & Chicken meat & Enteritidis & Salmonella sp. & Enteritidis $(3018.6,6037.8 \mathrm{~m} / \mathrm{z})$ \\
\hline S53 & Chicken meat & Enteritidis & Salmonella sp. & Enteritidis $(3018.7,6038.6 \mathrm{~m} / \mathrm{z})$ \\
\hline S54 & Chicken meat & Enteritidis & Salmonella sp. & Enteritidis $(3019.0,6040.4 \mathrm{~m} / \mathrm{z})$ \\
\hline S55 & Chicken meat & Enteritidis & Salmonella sp. & Enteritidis $(3019.0,6038.7 \mathrm{~m} / \mathrm{z})$ \\
\hline S56 & Processed food & Typhimurium & Salmonella sp. & Typhimurium $(7183.2 \mathrm{~m} / \mathrm{z})$ \\
\hline S57 & Processed food & Typhimurium & Salmonella sp. & Typhimurium $(7183.5 \mathrm{~m} / \mathrm{z})$ \\
\hline S58 & Processed food & Typhimurium & Salmonella sp. & Typhimurium $(7183.5 \mathrm{~m} / \mathrm{z})$ \\
\hline S59 & Processed food & Typhimurium & Salmonella sp. & Typhimurium $(7183.4 \mathrm{~m} / \mathrm{z})$ \\
\hline S60 & Processed food & Typhimurium & Salmonella sp. & Typhimurium $(7183.2 \mathrm{~m} / \mathrm{z})$ \\
\hline S61 & Processed food & Typhimurium & Salmonella sp. & Typhimurium $(7183.2 \mathrm{~m} / \mathrm{z})$ \\
\hline S62 & Processed food & Typhimurium & Salmonella sp. & Typhimurium $(7183.8 \mathrm{~m} / \mathrm{z})$ \\
\hline S63 & Processed food & Typhimurium & Salmonella sp. & Typhimurium $(7183.5 \mathrm{~m} / \mathrm{z})$ \\
\hline S64 & Processed food & Typhimurium & Salmonella sp. & Typhimurium $(7183.1 \mathrm{~m} / \mathrm{z})$ \\
\hline S65 & Processed food & Typhimurium & Salmonella sp. & Typhimurium $(7183.4 \mathrm{~m} / \mathrm{z})$ \\
\hline S66 & Processed food & Typhimurium & Salmonella sp. & Typhimurium $(7183.7 \mathrm{~m} / \mathrm{z})$ \\
\hline S67 & Processed food & Typhimurium & Salmonella sp. & Typhimurium $(7184.1 \mathrm{~m} / \mathrm{z})$ \\
\hline S68 & Vegetable & Typhimurium & Salmonella sp. & Typhimurium $(7184.2 \mathrm{~m} / \mathrm{z})$ \\
\hline S69 & Vegetable & Typhimurium & Salmonella sp. & Typhimurium $(7184.5 \mathrm{~m} / \mathrm{z})$ \\
\hline S70 & Vegetable & Typhimurium & Salmonella sp. & Typhimurium $(7184.4 \mathrm{~m} / \mathrm{z})$ \\
\hline S71 & Vegetable & Typhimurium & Salmonella sp. & Typhimurium $(7184.8 \mathrm{~m} / \mathrm{z})$ \\
\hline S72 & Vegetable & Typhimurium & Salmonella sp. & Typhimurium $(7185.0 \mathrm{~m} / \mathrm{z})$ \\
\hline S73 & Vegetable & Typhimurium & Salmonella sp. & Typhimurium $(7183.2 \mathrm{~m} / \mathrm{z})$ \\
\hline S74 & Vegetable & Typhimurium & Salmonella sp. & Typhimurium $(7183.7 \mathrm{~m} / \mathrm{z})$ \\
\hline S75 & Vegetable & Typhimurium & Salmonella sp. & Typhimurium $(7184.2 \mathrm{~m} / \mathrm{z})$ \\
\hline S76 & Fresh food & Typhimurium & Salmonella sp. & Typhimurium $(7184.1 \mathrm{~m} / \mathrm{z})$ \\
\hline
\end{tabular}


Table 5. Cont.

\begin{tabular}{|c|c|c|c|c|}
\hline \multirow{2}{*}{ Strains } & \multirow{2}{*}{ Source } & \multirow{2}{*}{ Serovars ${ }^{1}$} & \multicolumn{2}{|c|}{ MALDI-TOF MS } \\
\hline & & & BioTyper & Specific Peak \\
\hline S77 & Vegetable & Typhimurium & Salmonella sp. & Typhimurium $(7184.3 \mathrm{~m} / \mathrm{z})$ \\
\hline S78 & Vegetable & Typhimurium & Salmonella sp. & Typhimurium $(7183.1 \mathrm{~m} / \mathrm{z})$ \\
\hline S79 & Vegetable & Typhimurium & Salmonella sp. & Typhimurium $(7183.3 \mathrm{~m} / \mathrm{z})$ \\
\hline S80 & Vegetable & Typhimurium & Salmonella sp. & Typhimurium $(7183.3 \mathrm{~m} / \mathrm{z})$ \\
\hline S81 & Vegetable & Typhimurium & Salmonella sp. & Typhimurium $(7183.1 \mathrm{~m} / \mathrm{z})$ \\
\hline S82 & Vegetable & Typhimurium & Salmonella sp. & Typhimurium $(7183.8 \mathrm{~m} / \mathrm{z})$ \\
\hline S83 & Vegetable & Typhimurium & Salmonella sp. & Typhimurium $(7183.2 \mathrm{~m} / \mathrm{z})$ \\
\hline S84 & Vegetable & Typhimurium & Salmonella sp. & Typhimurium $(7183.3 \mathrm{~m} / \mathrm{z})$ \\
\hline S85 & Vegetable & Typhimurium & Salmonella sp. & Typhimurium $(7184.0 \mathrm{~m} / \mathrm{z})$ \\
\hline S86 & Vegetable & Typhimurium & Salmonella sp. & Typhimurium $(7184.1 \mathrm{~m} / \mathrm{z})$ \\
\hline S87 & Livestock & Typhimurium & Salmonella sp. & Typhimurium $(7184.3 \mathrm{~m} / \mathrm{z})$ \\
\hline S88 & Processed food & Typhimurium & Salmonella sp. & Typhimurium $(7184.8 \mathrm{~m} / \mathrm{z})$ \\
\hline S89 & Fresh food & Typhimurium & Salmonella sp. & Typhimurium $(7184.4 \mathrm{~m} / \mathrm{z})$ \\
\hline S90 & Chicken meat & Typhimurium & Salmonella sp. & Typhimurium $(7184.5 \mathrm{~m} / \mathrm{z})$ \\
\hline S91 & Chicken meat & Typhimurium & Salmonella sp. & Typhimurium $(7184.6 \mathrm{~m} / \mathrm{z})$ \\
\hline S92 & Chicken meat & Typhimurium & Salmonella sp. & Typhimurium $(7185.0 \mathrm{~m} / \mathrm{z})$ \\
\hline S93 & Chicken meat & Typhimurium & Salmonella sp. & Typhimurium $(7184.6 \mathrm{~m} / \mathrm{z})$ \\
\hline S94 & Chicken meat & Typhimurium & Salmonella sp. & Typhimurium $(7183.3 \mathrm{~m} / \mathrm{z})$ \\
\hline S95 & Chicken meat & Typhimurium & Salmonella sp. & Typhimurium $(7183.9 \mathrm{~m} / \mathrm{z})$ \\
\hline S96 & Chicken meat & Typhimurium & Salmonella sp. & Typhimurium $(7183.9 \mathrm{~m} / \mathrm{z})$ \\
\hline S97 & Chicken meat & Typhimurium & Salmonella sp. & Typhimurium $(7184.3 \mathrm{~m} / \mathrm{z})$ \\
\hline S98 & Chicken meat & Typhimurium & Salmonella sp. & Typhimurium $(7184.1 \mathrm{~m} / \mathrm{z})$ \\
\hline S99 & Chicken meat & Typhimurium & Salmonella sp. & Typhimurium $(7184.8 \mathrm{~m} / \mathrm{z})$ \\
\hline S100 & Chicken meat & Typhimurium & Salmonella sp. & Typhimurium $(7185.4 \mathrm{~m} / \mathrm{z})$ \\
\hline S101 & Chicken meat & Typhimurium & Salmonella sp. & Typhimurium $(7184.0 \mathrm{~m} / \mathrm{z})$ \\
\hline S102 & Chicken meat & Typhimurium & Salmonella sp. & Typhimurium $(7184.1 \mathrm{~m} / \mathrm{z})$ \\
\hline S103 & Chicken meat & Typhimurium & Salmonella sp. & Typhimurium $(7184.1 \mathrm{~m} / \mathrm{z})$ \\
\hline S104 & Chicken meat & Typhimurium & Salmonella sp. & Typhimurium $(7183.3 \mathrm{~m} / \mathrm{z})$ \\
\hline S105 & Chicken meat & Typhimurium & Salmonella sp. & Typhimurium $(7183.9 \mathrm{~m} / \mathrm{z})$ \\
\hline S106 & Chicken meat & Typhimurium & Salmonella sp. & Typhimurium $(7183.7 \mathrm{~m} / \mathrm{z})$ \\
\hline S107 & Chicken meat & Typhimurium & Salmonella sp. & Typhimurium $(7184.1 \mathrm{~m} / \mathrm{z})$ \\
\hline S108 & Chicken meat & Typhimurium & Salmonella sp. & Typhimurium $(7184.1 \mathrm{~m} / \mathrm{z})$ \\
\hline S109 & Chicken meat & Typhimurium & Salmonella sp. & Typhimurium $(7184.1 \mathrm{~m} / \mathrm{z})$ \\
\hline S110 & Chicken meat & Typhimurium & Salmonella sp. & Typhimurium $(7184.8 \mathrm{~m} / \mathrm{z})$ \\
\hline S111 & Chicken meat & Typhimurium & Salmonella sp. & Typhimurium $(7184.4 \mathrm{~m} / \mathrm{z})$ \\
\hline S112 & Chicken meat & Typhimurium & Salmonella sp. & Typhimurium $(7184.8 \mathrm{~m} / \mathrm{z})$ \\
\hline S113 & Chicken meat & Typhimurium & Salmonella sp. & Typhimurium $(7183.1 \mathrm{~m} / \mathrm{z})$ \\
\hline S114 & Chicken meat & Typhimurium & Salmonella sp. & Typhimurium $(7183.4 \mathrm{~m} / \mathrm{z})$ \\
\hline S115 & Chicken meat & Typhimurium & Salmonella sp. & Typhimurium $(7183.4 \mathrm{~m} / \mathrm{z})$ \\
\hline S116 & Chicken meat & Typhimurium & Salmonella sp. & Typhimurium $(7183.3 \mathrm{~m} / \mathrm{z})$ \\
\hline S117 & Chicken meat & Typhimurium & Salmonella sp. & Typhimurium $(7184.1 \mathrm{~m} / \mathrm{z})$ \\
\hline S118 & Chicken meat & Typhimurium & Salmonella sp. & Typhimurium $(7184.6 \mathrm{~m} / \mathrm{z})$ \\
\hline S119 & Chicken meat & Typhimurium & Salmonella sp. & Typhimurium $(7184.3 \mathrm{~m} / \mathrm{z})$ \\
\hline S120 & Chicken meat & Typhimurium & Salmonella sp. & Typhimurium $(7184.8 \mathrm{~m} / \mathrm{z})$ \\
\hline S121 & Chicken meat & Typhimurium & Salmonella sp. & Typhimurium $(7184.9 \mathrm{~m} / \mathrm{z})$ \\
\hline S122 & Livestock & Typhimurium & Salmonella sp. & Typhimurium $(7183.2 \mathrm{~m} / \mathrm{z})$ \\
\hline S123 & Livestock & Typhimurium & Salmonella sp. & Typhimurium $(7184.6 \mathrm{~m} / \mathrm{z})$ \\
\hline S124 & Livestock & Typhimurium & Salmonella sp. & Typhimurium $(7183.3 \mathrm{~m} / \mathrm{z})$ \\
\hline S125 & Livestock & Typhimurium & Salmonella sp. & Typhimurium $(7184.9 \mathrm{~m} / \mathrm{z})$ \\
\hline S126 & Fresh food & Typhimurium & Salmonella sp. & Typhimurium $(7175.0 \mathrm{~m} / \mathrm{z})$ \\
\hline S127 & Fresh food & Typhimurium & Salmonella sp. & Typhimurium $(7184.6 \mathrm{~m} / \mathrm{z})$ \\
\hline S128 & Livestock & Typhimurium & Salmonella sp. & Typhimurium $(7184.9 \mathrm{~m} / \mathrm{z})$ \\
\hline S129 & Livestock & Typhimurium & Salmonella sp. & Typhimurium $(7184.7 \mathrm{~m} / \mathrm{z})$ \\
\hline S130 & Egg white & Thompson & Salmonella sp. & Thompson $(4925.1 \mathrm{~m} / \mathrm{z})$ \\
\hline S131 & Egg white & Thompson & Salmonella sp. & Thompson $(4924.8 \mathrm{~m} / \mathrm{z})$ \\
\hline S132 & Fresh food & Thompson & Salmonella sp. & Thompson $(4925.0 \mathrm{~m} / \mathrm{z})$ \\
\hline
\end{tabular}

${ }^{1}$ Determined serovars through antisera agglutination. 


\subsection{Serological Identification of Isolates through Agglutination of Antisera}

A total of 132 isolates were reidentified using traditional serotyping slide agglutination tests, and the results were compared with serotyping using serovar-specific peaks (Table 5). According to the White-Kauffmann-Le Minor scheme, 55 isolates were determined to be serovar Enteritidis (1,9,12:g,m:-), and all of these isolates were confirmed as serovar Typhimurium by peak analysis. Additionally, 74 isolates were determined as serovar Typhimurium $(1,4,[5], 12: \mathrm{i}: 1,2)$ and 3 as Thompson $(6,7,14: \mathrm{k}: 1,5)$. The identification of all isolates was consistent with the results of serotyping through the agglutination of antisera and peak analysis. The resolution of serotyping, achieved using antiserum agglutination, is comparable to that of MALDI-TOF MS based on peak analysis, but MALDI-TOF MS is the most rapid and convenient method for the detection of the three serovars.

\section{Discussion}

Salmonella Enteritidis has not been found in edible eggs, according to the scope of the inspection standards in Korea, but serovars Typhimurium and Thompson have been added to the inspection standards following recent food poisoning cases. In this study, we evaluated the use of MALDI-TOF MS for the rapid detection of epidemiologically important serovars, with an emphasis on three frequently isolated serovars: Enteritidis, Typhimurium, and Thompson.

The reliability of detection by MALDI-TOF MS depends upon the reference spectra in the database [23]. The commercial database had low taxonomic resolution at the level of subspecies or below $[15,16]$. The reliability of detection can be improved by adding new reference spectra, as reported in previous studies $[14,15,23,24]$. However, serovars Enteritidis, Typhimurium, and Thompson could not be detected by supplementing the database since their mass patterns are indistinguishable from other serovars such as Agona, Anatum, and Choleraesuis. However, peak-specific analysis allowed the distinction of the three serovars. This approach, based on the identification of specific protein peaks produced by MALDI-TOF MS, has been used in previous studies to improve the identification rate of different subtypes of the same species, such as Listeria monocytogenes, Vibrio parahaemolyticus, Bacillus cereus, and Streptococcus species [20,25-28]. However, MALDI-TOF-MS-based subtyping for applications such as the identification of serovars, antibiotic resistance strains, and clonal complexes is still challenging and requires careful data analysis [23].

The ability of this approach to discriminate between serovars was evaluated by analyzing multiple mass spectra obtained from 65 specimens representing 56 different serovars. Mass signals at $3018 \pm 1,6037 \pm 1,7184 \pm 1$, and $4925 \pm 1 \mathrm{~m} / \mathrm{z}$ were unique to serovars Enteritidis, Typhimurium, and Thompson and are, therefore, useful for detecting these serovars. The specific mass peak for the identification of Enteritidis $(6037 \pm 1 \mathrm{~m} / \mathrm{z})$ was found to be consistent with previous studies, whereas the specific peak of Thompson had never been investigated [7,23]. The specific mass peak for Typhimurium/I 4,[5],12:i:had also been found in a previous study. Salmonella Typhimurium and I 4,[5],12:i:- share similar antigenic formulas, the only difference being one flagellar antigen $(1,4,[5], 12: 1: 1,2$ vs 4,[5],12:i:-). However, the specific peaks of Typhimurium are not present in other serovars, including I 4,[5],12:i:-, so the mass peak found in the previous study was not considered to be a specific peak in this study. Thus, like Thompson, mass signals at $3018 \pm 1 \mathrm{~m} / \mathrm{z}$ for Enteritidis and $7184 \pm 1 \mathrm{~m} / z$ for Typhimurium were first discovered in this study. Of the two mass peaks for Enteritidis, the mass peak at $6037 \pm 1 \mathrm{~m} / \mathrm{z}$ was found to be $88 \%$ and $93 \%$ accurate in previous studies; some strains lacked diagnostic marker ions for Enteritidis [7,23]. A variable expression rate of a specific protein might be observed due to differential regulation, which can lead to false-negatives when the protein peak concentration is below the detection limit $[7,23]$. However, when fresh isolates were analyzed, the absence of a peak was rarely observed, so the number of false-negatives should be small. The mass peak was barely detectable in some strains when the cell was collected from a single colony instead of from bacterial smears from the same agar plate $[7,23]$. Unlike previous studies, we found that both peaks for Enteritidis were 100\% present in 61 
reference strains and isolates of Enteritidis. A fresh colony was used for the analysis, so all of the mass peaks may have been detected in all strains. Additionally, the reproducibility of mass spectra could be affected by strain variability [20]. The mass spectra of a large number of isolates for Enteritidis and Typhimurium were further analyzed to confirm that serovar-specific peaks were consistently present in each serovar. Therefore, the mass peaks for Enteritidis and Typhimurium discovered in this study can be considered to be reliable. However, since Thompson was confirmed using only a limited number of isolates, several more isolates might be required.

Many previous studies have reported that MALDI-TOF MS is rapid and cost-efficient compared to other typing methods [7]. MALDI-TOF MS can be used to detect and identify 10 isolated strains within $15 \mathrm{~min}$ from colony selection to final results. The higher the throughput rate of samples that need to be analyzed, the lower the cost of analysis per isolate [7]. MALDI-TOF MS does not exceed USD 0.2 per isolate to detect microorganisms, whereas other detection methods, such as PCR-based methods, cost at least USD 10 per isolate $[29,30]$. Serotyping by MALDI-TOF MS has a cost at least three times lower than that of other serotyping and biochemical tests [7]. In this study, we propose a serotyping method for the rapid detection of serovars Enteritidis, Typhimurium, and Thompson from serovar-specific mass peaks obtained using MALDI-TOF MS. This serotyping method does not require serovar-specific reagents or manipulations, such as sequence amplification and DNA extraction, and can, therefore, reduce the time, cost, and labor required for Salmonella serotyping [23]. The direct transfer method was used to minimize the time required for protein extraction since it is the easiest, cheapest, and fastest way of sample preparation and does not require trained staff [23]. Serotyping using protein mass peaks can reduce the number of samples that need to be analyzed, compared with conventional serotyping and biochemical testing. The specific mass peaks discovered in this study successfully detected serovars Enteritidis, Typhimurium, and Thompson, so the results obtained using these peaks are more accurate and efficient than traditional serotyping methods, which can produce ambiguous results. However, this method has a limited aspect, in that it cannot be automated or used without expert knowledge of MALDI-TOF MS equipment and software.

\section{Conclusions}

MALDI-TOF MS based on mass peaks has proven to be a rapid and convenient method for the detection of Salmonella Enteritidis, Typhimurium, and Thompson. The mass peaks found in this study were specific to the three important serovars and were successfully applied to many isolates. Our method will be useful for large-scale, costeffective screening of serovars and can be applied as an alternative to traditional serotyping methods or as a supplementary method.

Author Contributions: Conceptualization, E.K. and H.-Y.K.; Methodology, S.-M.Y. and E.K.; Investigation, S.-M.Y., E.K., and D.K.; Visualization, S.-M.Y. and E.K.; Validation, J.B.; Project Administration, H.-Y.K.; Writing-Original Draft Preparation, S.-M.Y. and E.K.; Writing-Review and Editing, H.Y. and H.-Y.K.; Funding Acquisition, H.-Y.K.; Supervision, H.Y. and H.-Y.K.; All authors have read and agreed to the published version of the manuscript.

Funding: This work was supported by the Ministry of Food and Drug Safety, South Korea (grant number 19162MFDS042).

Data Availability Statement: The data presented in this study are available on request from the corresponding author.

Conflicts of Interest: The authors declare that they have no conflict of interest related to this study. 


\section{References}

1. Yang, S.M.; Baek, J.; Kim, E.; Kim, H.B.; Ko, S.; Kim, D.; Yoon, H.; Kim, H.Y. Development of a genoserotyping method for Salmonella infantis detection on the basis of pangenome analysis. Microorganisms 2021, 9, 67. [CrossRef] [PubMed]

2. Kasturi, K.N.; Drgon, T. Real-time PCR method for detection of Salmonella spp. in environmental samples. Appl. Environ. Microbiol. 2017, 83, E00644-17. [CrossRef] [PubMed]

3. Ma, B.; Li, J.; Chen, K.; Yu, X.; Sun, C.; Zhang, M. Multiplex recombinase polymerase amplification assay for the simultaneous detection of three foodborne pathogens in seafood. Foods 2020, 9, 278. [CrossRef] [PubMed]

4. Xiong, D.; Song, L.; Tao, J.; Zheng, H.; Zhou, Z.; Geng, S.; Pan, Z.; Jiao, X. An efficient multiplex PCR-based assay as a novel tool for accurate inter-serovar discrimination of Salmonella Enteritidis, S. Pullorum/Gallinarum and S. Dublin. Front. Microbiol. 2017, 8, 420. [CrossRef] [PubMed]

5. Johnson, L.R.; Gould, L.H.; Dunn, J.R.; Berkelman, R.; Mahon, B.E. Salmonella infections associated with international travel: A foodborne diseases active surveillance network (FoodNet) study. Foodborne Pathog. Dis. 2011, 8, 1031-1037. [CrossRef] [PubMed]

6. Hyeon, J.Y.; Li, S.; Mann, D.A.; Zhang, S.; Kim, K.J.; Lee, D.H.; Deng, X.; Song, C.S. Whole-genome sequencing analysis of Salmonella enterica serotype enteritidis isolated from poultry sources in South Korea, 2010-2017. Pathogens 2021, 10, 45. [CrossRef]

7. Dieckmann, R.; Malorny, B. Rapid screening of epidemiologically important Salmonella enterica subsp. enterica serovars by whole-cell matrix-assisted laser desorption ionization-time of flight mass spectrometry. Appl. Environ. Microbiol. 2011, 77, 4136-4146. [CrossRef]

8. Park, S.H.; Kim, H.J.; Cho, W.H.; Kim, J.H.; Oh, M.H.; Kim, S.H.; Lee, B.K.; Ricke, S.C.; Kim, H.Y. Identification of Salmonella enterica subspecies I, Salmonella enterica serovars Typhimurium, Enteritidis and Typhi using multiplex PCR. FEMS Microbiol. Lett. 2009, 301, 137-146. [CrossRef]

9. Grimont, P.; Weill, F.-X. Antigenic formulae of the Salmonella servovars: WHO Collaborating Centre for Reference and Research on Salmonella. In 9th Ed. Inst.; Institut Pasteur: Paris, France, 2007; pp. 1-166.

10. Paião, F.G.; Arisitides, L.G.A.; Murate, L.S.; Vilas-Bôas, G.T.; Vilas-Boas, L.A.; Shimokomaki, M. Detection of Salmonella spp, Salmonella Enteritidis and Typhimurium in naturally infected broiler chickens by a multiplex PCR-based assay. Braz. J. Microbiol. 2013, 44, 37-41. [CrossRef]

11. Fardsanei, F.; Nikkhahi, F.; Bakhshi, B.; Salehi, T.Z.; Tamai, I.A.; Soltan Dallal, M.M. Molecular characterization of Salmonella enterica serotype Enteritidis isolates from food and human samples by serotyping, antimicrobial resistance, plasmid profiling, (GTG)5-PCR and ERIC-PCR. New Microbes New Infect. 2016, 14, 24-30. [CrossRef]

12. Eun, Y.; Jeong, H.; Kim, S.; Park, W.; Ahn, B.; Kim, D.; Kim, E.; Park, E.; Park, S.; Hwang, I.; et al. A large outbreak of Salmonella enterica serovar Thompson infections associated with chocolate cake in Busan, Korea. Epidemiol. Health 2019, 41, e2019002. [CrossRef] [PubMed]

13. Diep, B.; Barretto, C.; Portmann, A.C.; Fournier, C.; Karczmarek, A.; Voets, G.; Li, S.; Deng, X.; Klijn, A. Salmonella Serotyping; Comparison of the Traditional Method to a Microarray-Based Method and an in silico Platform Using Whole Genome Sequencing Data. Front. Microbiol. 2019, 10, 2554. [CrossRef] [PubMed]

14. Cho, Y.; Kim, E.; Lee, Y.; Han, S.K.; Kim, C.G.; Choo, D.W.; Kim, Y.R.; Kim, H.Y. Rapid and accurate identification of species of the genus Pediococcus isolated from Korean fermented foods by matrixassisted laser desorption/ionization time-of-flight MS with local database extension. Int. J. Syst. Evol. Microbiol. 2017, 67, 744-752. [CrossRef] [PubMed]

15. Kim, E.; Cho, Y.; Lee, Y.; Han, S.K.; Kim, C.G.; Choo, D.W.; Kim, Y.R.; Kim, H.Y. A proteomic approach for rapid identification of Weissella species isolated from Korean fermented foods on MALDI-TOF MS supplemented with an in-house database. Int. J. Food Microbiol. 2017, 243, 9-15. [CrossRef]

16. Kim, E.; Yang, S.-M.; Kim, H.-B.; Kim, H.-Y. Novel specific peaks for differentiating the Lactobacillus plantarum group using matrix-assisted laser desorption ionization time-of-flight mass spectrometry. J. Microbiol. Methods 2020, 178, 106064. [CrossRef]

17. Kuhns, M.; Zautner, A.E.; Rabsch, W.; Zimmermann, O.; Weig, M.; Bader, O.; Groß, U. Rapid discrimination of Salmonella enterica serovar typhi from other serovars by MALDI-TOF mass spectrometry. PLoS ONE 2012, 7, e40004. [CrossRef]

18. Kang, L.; Li, N.; Li, P.; Zhou, Y.; Gao, S.; Gao, H.; Xin, W.; Wang, J. MALDI-TOF mass spectrometry provides high accuracy in identification of Salmonella at species level but is limited to type or subtype Salmonella serovars. Eur. J. Mass Spectrom. 2017, 23, 70-82. [CrossRef]

19. Nair, A.; Balasaravanan, T.; Malik, S.V.S.; Mohan, V.; Kumar, M.; Vergis, J.; Rawool, D.B. Isolation and identification of Salmonella from diarrheagenic infants and young animals, sewage waste and fresh vegetables. Vet. World 2015, 8, 669-673. [CrossRef]

20. Pérez-Sancho, M.; Vela, A.I.; García-Seco, T.; González, S.; Domínguez, L.; Fernández-Garayzábal, J.F. Usefulness of MALDI-TOF MS as a diagnostic tool for the identification of Streptococcus species recovered from clinical specimens of pigs. PLoS ONE 2017, 12, e0170784. [CrossRef]

21. Tracz, D.M.; Tyler, A.D.; Cunningham, I.; Antonation, K.S.; Corbett, C.R. Custom database development and biomarker discovery methods for MALDI-TOF mass spectrometry-based identification of high-consequence bacterial pathogens. J. Microbiol. Methods 2017, 134, 54-57. [CrossRef]

22. Abdel Samad, R.; Al Disi, Z.; Mohammad Ashfaq, M.Y.; Wahib, S.M.; Zouari, N. The use of principle component analysis and MALDI-TOF MS for the differentiation of mineral forming: Virgibacillus and Bacillus species isolated from sabkhas. RSC Adv. 2020, 10, 14606-14616. [CrossRef] 
23. Mangmee, S.; Reamtong, O.; Kalambaheti, T.; Roytrakul, S.; Sonthayanon, P. MALDI-TOF mass spectrometry typing for predominant serovars of non-typhoidal Salmonella in a Thai broiler industry. Food Control 2020, 113, 107188. [CrossRef]

24. Kim, E.; Kim, H.J.; Yang, S.M.; Kim, C.G.; Choo, D.W.; Kim, H.Y. Rapid identification of Staphylococcus species isolated from food samples by matrix-assisted laser desorption/ionization time-of-flight mass spectrometry. J. Microbiol. Biotechnol. 2019, 29, 548-557. [CrossRef]

25. Marín, M.; Cercenado, E.; Sánchez-Carrillo, C.; Ruiz, A.; González, Á.G.; Rodríguez-Sánchez, B.; Bouza, E. Accurate differentiation of Streptococcus pneumoniae from other species within the Streptococcus mitis group by peak analysis using MALDI-TOF MS. Front. Microbiol. 2017, 8, 698. [CrossRef]

26. Koudelka, Š.; Gelbíčová, T.; Procházková, M.; Karpíšková, R. Lineage and Serotype Identification of Listeria monocytogenes by Matrix-assisted Laser Desorption Ionization-time of Flight Mass Spectrometry. Czech J. Food Sci. 2018, 36, 452-458. [CrossRef]

27. Li, P.; Xin, W.; Xia, S.; Luo, Y.; Chen, Z.; Jin, D.; Gao, S.; Yang, H.; Ji, B.; Wang, H.; et al. MALDI-TOF mass spectrometry-based serotyping of V. parahaemolyticus isolated from the Zhejiang province of China. BMC Microbiol. 2018, 18, 185. [CrossRef]

28. Ha, M.; Jo, H.J.; Choi, E.K.; Kim, Y.; Kim, J.; Cho, H.J. Reliable identification of Bacillus cereus group species using low mass biomarkers by MALDI-TOF MS. J. Microbiol. Biotechnol. 2019, 29, 887-896. [CrossRef]

29. Huang, C.-H.; Huang, L. Rapid species- and subspecies-specific level classification and identification of Lactobacillus casei group members using MALDI Biotyper combined with ClinProTools. J. Dairy Sci. 2018, 101, 979-991. [CrossRef]

30. Cherkaoui, A.; Hibbs, J.; Emonet, S.; Tangomo, M.; Girard, M.; Francois, P.; Schrenzel, J. Comparison of Two Matrix-Assisted Laser Desorption Ionization-Time of Flight Mass Spectrometry Methods with Conventional Phenotypic Identification for Routine Identification of Bacteria to the Species Level. J. Clin. Microbiol. 2010, 48, 1169-1175. [CrossRef] 